Article

\title{
An Approach to Characterizing the Complicated Sequential Metabolism of Salidroside in Rats
}

\author{
Zhiqiang Luo ${ }^{1,+}$, Xiaoyun Ma ${ }^{1,+}$, Yang Liu ${ }^{1, *}$, Lina Lu ${ }^{1}$, Ruirui Yang ${ }^{1}$, Guohua Yu ${ }^{1}$, \\ Mohan Sun ${ }^{1}$, Shaokun Xin ${ }^{2}$, Simin Tian ${ }^{1}$, Xinjing Chen ${ }^{1}$ and Haiyu Zhao ${ }^{3, *}$ \\ 1 School of Chinese Materia Medica, Beijing University of Chinese Medicine, Beijing 100102, China; \\ lzq4y3r@126.com (Z.L.); sijidehuaiye@126.com (X.M.); luludc399@163.com (L.L.); \\ yangrr5021@126.com (R.Y.); sufei_sophie@163.com (G.Y.); m13001147155@163.com (M.S.); \\ tiansimin1990@163.com (S.T.); chxj9208@sina.com (X.C.) \\ 2 School of Traditional Chinese Medicine, Capital Medical University, Beijing 100069, China; \\ xyyzxxx@163.com \\ 3 Institute of Chinese Materia Medica, China Academy of Chinese Medical Sciences, Beijing 100700, China \\ * Correspondence: liuyang@bucm.edu.cn (Y.L.); zhyzhy007@gmail.com (H.Z.); \\ Tel./Fax: +86-010-8473-8611 (Y.L.) \\ + These authors contributed equally to this work.
}

Academic Editor: Derek J. McPhee

Received: 11 April 2016; Accepted: 25 May 2016; Published: 30 May 2016

\begin{abstract}
Metabolic study of bioactive compounds that undergo a dynamic and sequential process of metabolism is still a great challenge. Salidroside, one of the most active ingredients of Rhodiola crenulata, can be metabolized in different sites before being absorbed into the systemic blood stream. This study proposed an approach for describing the sequential biotransformation process of salidroside based on comparative analysis. In vitro incubation, in situ closed-loop and in vivo blood sampling were used to determine the relative contribution of each site to the total metabolism of salidroside. The results showed that salidroside was stable in digestive juice, and it was metabolized primarily by the liver and the intestinal flora and to a lesser extent by the gut wall. The sequential metabolism method described in this study could be a general approach to characterizing the metabolic routes in the digestive system for natural products.
\end{abstract}

Keywords: salidroside; sequential metabolism; in situ closed-loop

\section{Introduction}

Salidroside ( $p$-hydroxyphenethyl-beta-D-glucoside), a compound with a chemical structure of phenol glycosides (Figure 1), has been identified as one of the most potent ingredients isolated from Rhodiola crenulata [1]. Salidroside reportedly possesses broad pharmacological activities, such as resisting anoxia [2], anti-fatigue [3], anti-inflammation [4,5], anti-aging [6], anti-cancer [7-9], antioxidation [10], cardioprotective [11], and hepatoprotective [12] properties. As a well-known natural product, salidroside is widely used as a bioactive ingredient for functional foods, dietary supplements, and phytomedicines around the world [13-16].

Although salidroside exhibited a wide variety of biological activities in in vitro and in vivo experiments, only a few reports regarding the metabolism and disposition of salidroside are available. Han et al. developed a LC/MS method to identify salidroside and its major metabolites in rat plasma, bile, urine and feces, and up to 7 metabolites were detected. Among them, sulfate and glucuronidation conjugation were the primary pathway for salidroside [17]. Hu et al. identified eight metabolites of salidroside from the rat urine samples via ultra-performance liquid chromatography coupled with quadrupole time-of-flight mass spectrometry and high performance liquid chromatography 
coupled with quadrupole-linear ion trap mass spectrometry [18]. Guo et al. studied the metabolism of salidroside to its aglycone $p$-Tyrosol in rats [19]. However, the existing results could not provide a comprehensive map of the dynamic biotransformation process of salidroside following oral administration. Accordingly, it is necessary to study the sequential metabolic changes of salidroside in vivo.<smiles>OCC1OC(OCCc2ccc(O)cc2)C(O)C(O)C1O</smiles>

Figure 1. Chemical structure of salidroside.

Hence, the primary goal of this study was to develop a holistic, sequential and reproducible approach to describe the complicated sequential metabolism of salidroside before absorption into the systemic blood stream. In brief, the approach includes four fundamental steps: (1) enzymatic transformation in digestive juice, (2) biotransformation by intestinal flora tract, (3) gut wall metabolism, and (4) hepatic metabolism [20]. The approach was based on comparative analysis and a carefully designed mix of metabolic techniques such as in vitro incubation, in situ closed-loop and in vivo blood sampling. Ultra-high-pressure liquid chromatography coupled with linear ion trap-Orbitrap tandem mass spectrometry (UHPLC-LTQ-Orbitrap) was used to analyze the metabolites of salidroside in different metabolic sites. High-resolution mass spectrometry such as LTQ-Orbitrap plays a crucial role in metabolic analyses owing to its accurate MS data and reliable metabolite identification when attempting to discriminate metabolites with different MS/MS fragments [21]. By the results, we can determine the probable metabolic pathways of salidroside and the sequential contribution of each metabolic site. Research on the sequential metabolism of salidroside would provide helpful information on how the biological activities of these components are changed before these components are delivered to the target sites.

\section{Results and Discussion}

\subsection{Stability of Salidroside in Artificial Gastric Juice and Intestinal Juice}

Salidroside was quite stable in artificial gastric juice and intestinal juice. The content of salidroside was not altered significantly according to the peak areas during in vitro incubation with digestive juices $(\mathrm{RSD}<5.0 \%)$.

\subsection{Fragmentation of Salidroside Standard}

The chromatographic and mass spectrometric conditions were optimized using salidroside standard. Metabolite characterization was on the basis of the fragmentation patterns of salidroside.

As shown in Figure 2A, the deprotonated molecular ion of salidroside at $\mathrm{m} / \mathrm{z} 299.1126$ gave rise to six major product ions at $m / z 113.0242,119.0348,131.0346,143.0347,161.0451,179.0556$. Two important fragmentations from deprotonated salidroside were the cleavage of the glycosidic linkage leading to the formation of $m / z 179.0556$ and 119.0348. The fragment at $m / z 161.0451$ was generated through the loss of $\mathrm{H}_{2} \mathrm{O}$ from the ion at $m / z$ 179.0556, and then produced the ion at $m / z 143.0347$ by the further elimination of $\mathrm{H}_{2} \mathrm{O}$. The fragment ion at $m / z 131.0346$ was rationalized to originate from the product ion at $m / z 179.0556$ through the loss of one molecule of $\mathrm{CH}_{2} \mathrm{O}$ and one molecule of $\mathrm{H}_{2} \mathrm{O}$. Subsequently, the predominant fragmentation ion at $m / z 113.0242$ was produced by the reduction of $\mathrm{H}_{2} \mathrm{O}$ from $m / z$ 131.0346. Thus, it can be concluded that the ions at $m / z 113.0242,119.0347,131.0346,143.0347,161.0451$, 179.0556 were the characteristic product ions of salidroside. 

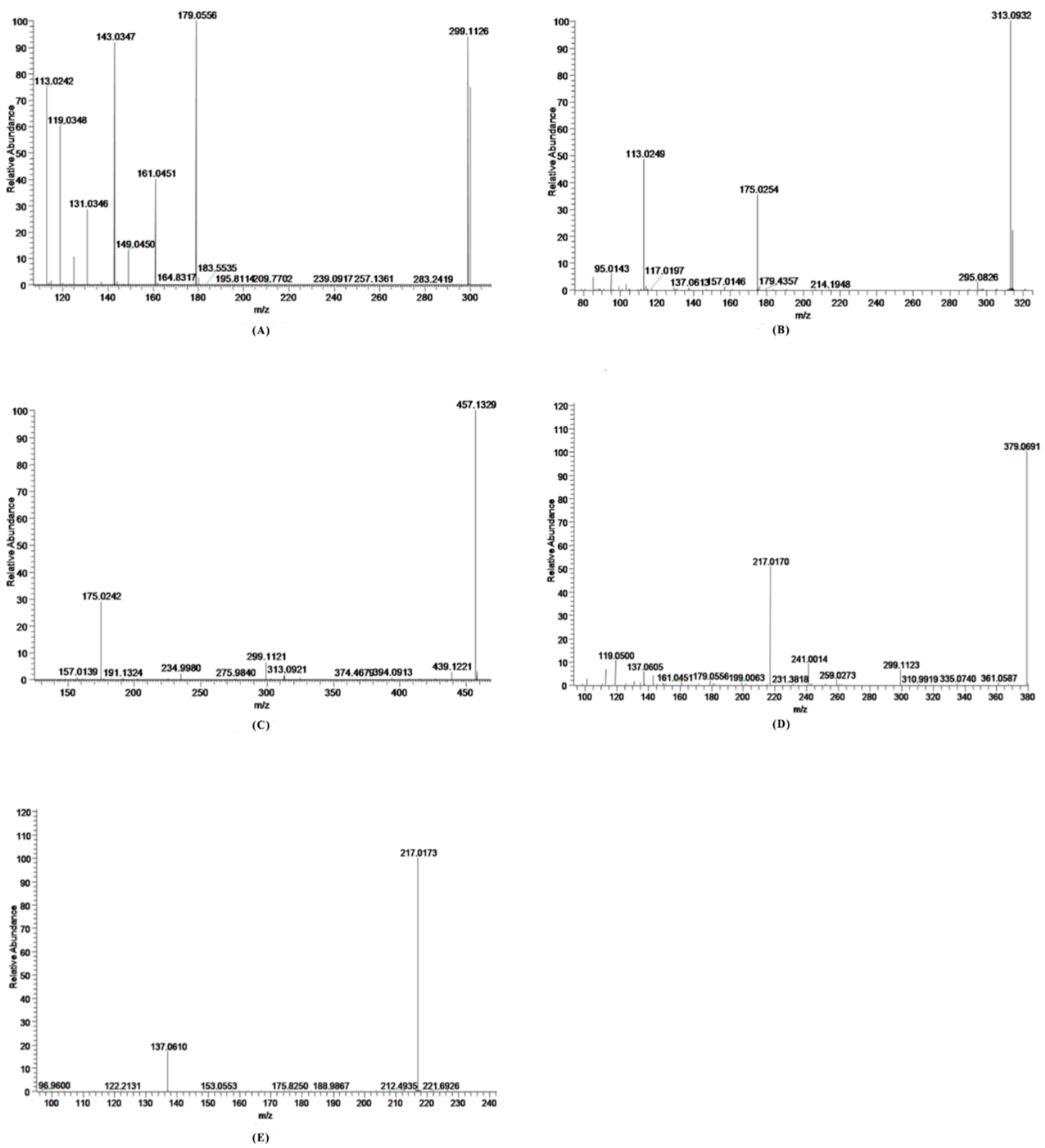

Figure 2. Representative MS/MS spectrum: (A) M0 ( $\mathrm{m} / \mathrm{z} 299) ;$ (B) M1 (m/z 313); (C) M2 (m/z 475); (D) M3 ( $m / z$ 379); (E) M4 ( $m / z$ 217).

\subsection{Identification of Salidroside Metabolites in Different Plasma Samples}

Metabolites of salidroside were detected and identified by comparing plasma samples from salidroside-treated rats with corresponding control samples. The structures of the metabolites were elucidated by comparing their molecular weights and product ions with those of the parent drug [22]. A total of 4 metabolites were found from different plasma samples. Detailed information on salidroside (M0) and its metabolites (M1-M4) was summarized in Table 1. 
Table 1. UHPLC-LTQ-Orbitrap analysis and relative percentage area of salidroside and four metabolites in different blood samples.

\begin{tabular}{|c|c|c|c|c|c|c|c|c|c|c|c|}
\hline \multirow{2}{*}{ Metabolite } & \multirow{2}{*}{$t_{R} / \min$} & \multirow{2}{*}{ Found (Da) } & \multirow{2}{*}{ Calculated (Da) } & \multirow{2}{*}{ Error (ppm) } & \multirow{2}{*}{$\begin{array}{l}\text { Fragment ion } \\
\quad m / z \text { (Da) }\end{array}$} & \multirow{2}{*}{ Formula } & \multirow{2}{*}{$\begin{array}{l}\text { Metabolite } \\
\text { Description }\end{array}$} & \multicolumn{4}{|c|}{ Relative Percentage Area in Different Blood Samples ${ }^{1}$} \\
\hline & & & & & & & & GWM $^{2}$ & WOIF & WIIF & HM \\
\hline M0 & 11.17 & 299.1126 & 299.1125 & 0.4 & $\begin{array}{l}179.0556 \\
119.0348 \\
161.0451 \\
143.0347 \\
131.0346 \\
113.0242\end{array}$ & $\mathrm{C}_{14} \mathrm{H}_{20} \mathrm{O}_{7}$ & Parent & $94.26 \%$ & $99.59 \%$ & $43.85 \%$ & $22.93 \%$ \\
\hline M1 & 8.32 & 313.0932 & 313.0918 & 4.5 & $\begin{array}{l}175.0254 \\
113.0249 \\
295.0826\end{array}$ & $\mathrm{C}_{14} \mathrm{H}_{18} \mathrm{O}_{8}$ & $\begin{array}{l}\text { Deglycosylation + } \\
\text { Glucuronidation }\end{array}$ & $2.17 \%$ & $0.38 \%$ & $15.67 \%$ & $7.43 \%$ \\
\hline M2 & 8.93 & 475.1464 & 475.1446 & 3.8 & $\begin{array}{l}457.1329 \\
175.0242 \\
299.1121\end{array}$ & $\mathrm{C}_{20} \mathrm{H}_{28} \mathrm{O}_{13}$ & Glucuronidation & $0.66 \%$ & $0.04 \%$ & $3.52 \%$ & $16.42 \%$ \\
\hline M3 & 10.80 & 379.0691 & 379.0693 & -0.6 & $\begin{array}{l}217.0170 \\
299.1123 \\
137.0605 \\
119.0500\end{array}$ & $\mathrm{C}_{14} \mathrm{H}_{20} \mathrm{O}_{10} \mathrm{~S}$ & Sulfation & $2.91 \%$ & ND & $7.00 \%$ & $11.00 \%$ \\
\hline M4 & 11.61 & 217.0173 & 217.0165 & 3.4 & 137.0610 & $\mathrm{C}_{8} \mathrm{H}_{9} \mathrm{O}_{5} \mathrm{~S}$ & $\begin{array}{c}\text { Deglycosylation + } \\
\text { Sulfation }\end{array}$ & $\mathrm{ND}^{3}$ & ND & $29.96 \%$ & $42.23 \%$ \\
\hline
\end{tabular}

${ }^{1}$ The relative percentage area was calculated as (peak area of each metabolite/total
group; WIIF, within intestinal flora group; HM, hepatic metabolism; ${ }^{3}$ Not detected. 


\subsubsection{Parent Compound M0}

Salidroside (M0) was identified according to the UHPLC retention time (11.17 min), the accurate MS at $m / z 299.1126[\mathrm{M}-\mathrm{H}]^{-}$, and MS/MS spectra from the authentic standard. Characteristic fragment ions at $m / z 113,119,131,143,161,179$ could also be found in the MS/MS spectra.

\subsubsection{Metabolite M1}

M1 (retention time $t_{R}=8.32 \mathrm{~min}$ ) showed the molecular ion at $m / z 313.0932(4.5 \mathrm{ppm}$, elemental composition of $\mathrm{C}_{14} \mathrm{H}_{17} \mathrm{O}_{8}$ ), which was 176 Da higher than the $m / z$ of the aglycone $p$-tyrosol, suggesting a glucuronic acid group was conjugated to the aglycone $p$-tyrosol [18]. According to the MS/MS spectrum (Figure 2B), two major product ions of M1 were found at $m / z 175.0254$ (the $[\mathrm{M}-\mathrm{H}]^{-}$ion of glucuronic acid) and $113.0249\left(\mathrm{C}_{5} \mathrm{H}_{5} \mathrm{O}_{3}\right)$. The fragment at $m / z 295.0826$ was generated through the loss of $\mathrm{H}_{2} \mathrm{O}$ from the quasi-molecular ion.

\subsubsection{Metabolite M2}

M2 (retention time $\left.t_{\mathrm{R}}=8.93 \mathrm{~min}\right)$ displayed an $[\mathrm{M}-\mathrm{H}]^{-}$ion at $\mathrm{m} / z 475.1464(3.8 \mathrm{ppm}$, elemental composition of $\mathrm{C}_{20} \mathrm{H}_{27} \mathrm{O}_{13}$ ), which was 176 Da higher than the $m / z$ of $\mathrm{M} 0$, indicating that it was the glucuronide conjugated product of salidroside [18]. The major fragment ion (Figure 2C) at $m / z 457.1329$ was a $\mathrm{H}_{2} \mathrm{O}$ loss from the quasi-molecular ion. The product ions at $m / z 175.0242$ and 299.1121 were ions of glucuronic acid and salidroside produced by M2.

\subsubsection{Metabolite M3}

M3 (retention time $t_{\mathrm{R}}=10.80 \mathrm{~min}$ ) exhibited a $[\mathrm{M}-\mathrm{H}]^{-}$ion at $m / z 379.0691(-0.6 \mathrm{ppm}$, elemental composition of $\mathrm{C}_{14} \mathrm{H}_{19} \mathrm{O}_{10} \mathrm{~S}$ ), which was 80 Da heavier than that of salidroside, indicating sulfation of salidroside [18]. The major fragment ion determined at $m / z 217.0170$ was shaped by the elimination of Glu (162 Da). Characteristic product ions at $m / z$ 299, 137, 119 were also found in the MS $^{2}$ fragmentation (Figure 2D).

\subsubsection{Metabolite M4}

M4 (retention time $t_{R}=11.61 \mathrm{~min}$ ) exhibited a $[\mathrm{M}-\mathrm{H}]^{-}$ion at $m / z 217.0173(3.4 \mathrm{ppm}$, elemental composition of $\mathrm{C}_{8} \mathrm{H}_{9} \mathrm{O}_{5} \mathrm{~S}$ ), which was 80 Da heavier than that of the aglycone $p$-tyrosol, indicating a sulfuric acid group was M4 [18]. The major fragment ion (Figure 2E) at $\mathrm{m} / z$ 137.0610 was the aglycone $p$-tyrosol.

\subsection{The Sequential Process of Salidroside in the Digestive System}

Using the sequential metabolism method, a total of 4 metabolites of salidroside have been detected and identified in different plasma samples. Our results indicated that glucuronidation, sulfation, and deglycosylation were the major metabolic pathways of salidroside. The contribution of each metabolic site to the overall metabolism was also shown in Table 1. When salidroside was administered orally, enzymes in the gut wall may play an important role in the initial metabolism. However, results showed that the biotransformation rate of salidroside by the gut wall was very low. It can be noted that intestinal flora tract and liver were the major metabolic sites for salidroside. Salidroside appears to undergo sequential and multiple pathways of degradation and metabolism by intestinal flora tract and liver to produce active metabolites or intermediates, thereby exerting in vivo pharmacological functions. The sequential process of salidroside in the digestive system is shown in Figure 3. 


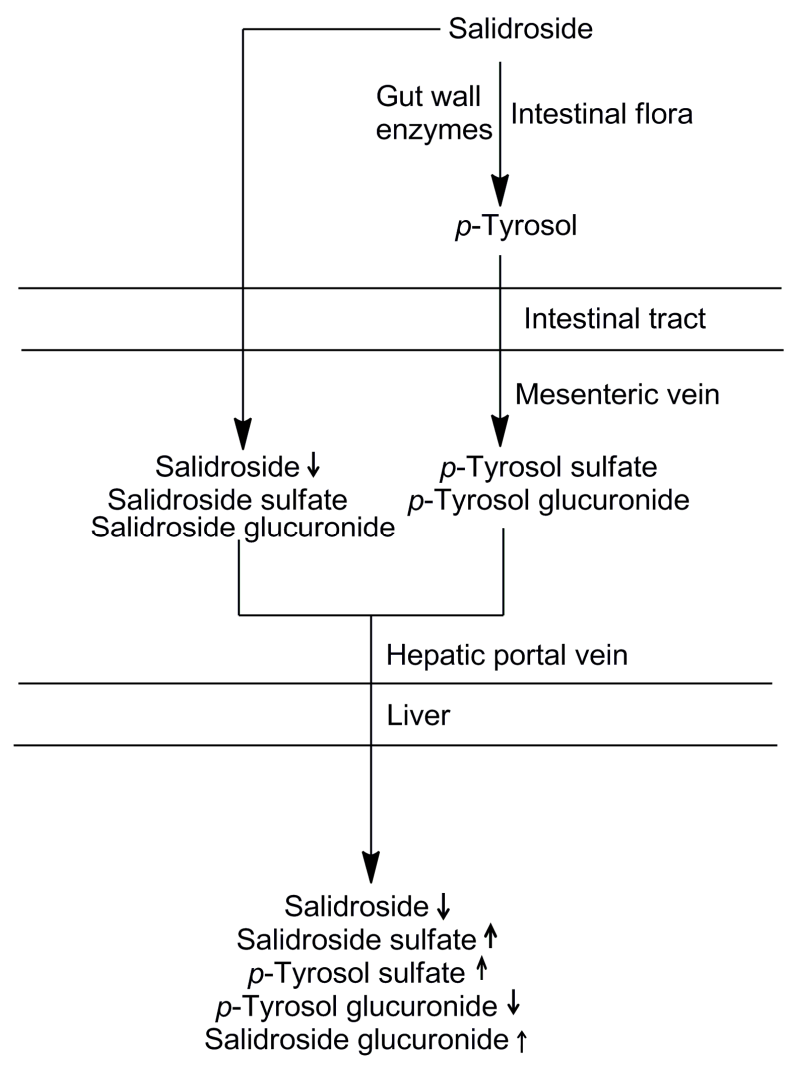

Figure 3. The sequential process of salidroside in the digestive system.

\section{Experimental Section}

\subsection{Chemicals and Reagents}

Salidroside (purity $>98 \%$ ) was purchased from Nanjing Zelang Biological Technology Co., Ltd. (Jiangsu, China). HPLC-grade acetonitrile and formic acid were obtained from Fisher Scientific. High purity water was prepared by using the Millipore Milli Q plus purification system. All other solvents and chemicals used were of analytical grade and commercially available.

The perfusion solution was Krebs-Ringer (K-R) buffer solution, which contained $7.8 \mathrm{~g} \mathrm{NaCl}$, $0.35 \mathrm{~g} \mathrm{KCl}, 1.37 \mathrm{~g} \mathrm{NaHCO}_{3}, 0.02 \mathrm{~g} \mathrm{MgCl}_{2}, 0.32 \mathrm{~g} \mathrm{NaH}_{2} \mathrm{PO}_{4}$, and $1.48 \mathrm{~g}$ glucose in $1000 \mathrm{~mL}$ distilled water.

\subsection{Preparation Perfusion Solution of Salidroside}

The perfusion solution of $10 \mathrm{mg} / \mathrm{mL}$ salidroside was prepared by dissolving $500 \mathrm{mg}$ salidroside in $50 \mathrm{~mL} \mathrm{K-R}$ buffer solution.

\subsection{Animals}

Sprague-Dawley rats (male, 200-250 g) were obtained from Spfanimals Laboratory Animal Technology Co. Ltd (Beijing, China). All animal experiments were conducted using protocols approved by the University Committee on Ethics in the Care and Use of Laboratory Animals (SYXKJING(2001-0024)), and the animals were housed and handled according to the Laboratory Animal Medicine Guidelines of Beijing University of Chinese Medicine. Before the study, the rats were kept in a temperature- and humidity-controlled room $\left(23^{\circ} \mathrm{C}, 60 \%\right.$ air humidity) with unlimited access to standard diet and water. The rats were acclimated for at least 7 days, then fasted overnight but supplied with water ad libitum prior to the date of the experiment. 


\subsection{Stability in Digestive Juice with Enzymes}

Salidroside $(1 \mathrm{mg})$ was added into $15 \mathrm{~mL}$ of artificial gastric juice $(0.08 \mathrm{M} \mathrm{HCl}$ containing $50 \mathrm{mg}$ of pepsin, $\mathrm{pH} 1.5$ ) and incubated in a $37^{\circ} \mathrm{C}$ shaking water bath for $0.5,2$, or $4 \mathrm{~h}$, respectively. The reactions were rapidly terminated by water-saturated $n$-butanol. The mixture was centrifuged at $5000 \times g$ for $15 \mathrm{~min}$, and then the supernatant was evaporated to dryness under a stream of nitrogen. The residue was dissolved in $500 \mu \mathrm{L}$ of methanol, and then filtered through a $0.45 \mu \mathrm{m}$ membrane for HPLC analysis.

Salidroside $(1 \mathrm{mg})$ was added into $15 \mathrm{~mL}$ of artificial intestinal juice $\left(0.05 \mathrm{M} \mathrm{KH}_{2} \mathrm{PO}_{4}\right.$ containing $50 \mathrm{mg}$ of pancreatin, $\mathrm{pH}$ 6.8) and incubated in a $37^{\circ} \mathrm{C}$ shaking water bath for 1,3 , or $6 \mathrm{~h}$, respectively. The samples were prepared as the procedure described in artificial gastric juice.

\subsection{Intestinal Flora Metabolism Method}

The in situ closed-loop method was extensively used to study intestinal absorption. The approach enables intestinal absorption to occur at body temperature for an appointed time. The model also allows absorption to be measured separately at different regions of rat intestine, jejunum, ileum and colon [23].

The complex surgical procedure and the in situ closed-loop experiments were conducted in accordance with the methods described previously $[20,23]$. The rats were divided into two groups. In one group, blood was collected from the mesenteric vein and the drug was injected into the gut loop directly. In the other group, blood was also collected from the mesenteric vein, but the drug was injected only after the intestinal flora had been flushed out.

Before perfusion surgical operation, five to seven rats were selected for donor blood per experiment and a total of 50-70 $\mathrm{mL}$ blood was obtained from the abdominal aorta with a heparinized syringe. The blood incubated in a $37^{\circ} \mathrm{C}$ water bath was prepared to be transfused into the recipient rat through the jugular vein to balance the blood loss via the mesenteric vein. The recipient rat was anesthetized with chloral hydrate $(400 \mathrm{mg} / \mathrm{kg}$ ) and placed under a heat lamp to maintain normal body temperature. To sustain the anesthetic condition, one third of the initial dose of chloral hydrate was administered in the following experiment.

Upon verification of the loss of pain reflex, the left external jugular vein was exposed and cannulated with a 24 GBD Intima II catheter (Becton Dickinson Medical Devices Co. Ltd, Beijing, China) to transfuse blood collected previously from donor rats. Then the abdomen was opened with a midline incision and a $5 \mathrm{~cm}$ colon segment was isolated by two ligatures to form a loop. To collect venous outflow, a $24 \mathrm{G}$ catheter with heparinized saline was inserted into the mesenteric vein draining the segment. The blood flow amounted to $0.3 \mathrm{~mL} / \mathrm{min}$ to avoid blood loss.

For the study without intestinal flora, the intestinal content was washed out by perfusing the loop with prewarmed K-R buffer solution. When the flora was carefully expelled, air was pumped through the syringe to clean the lumen. Salidroside solution $(2 \mathrm{~mL})$ was injected into a loop with a syringe. At the conclusion of surgery, the loop of gut with blood supply intact was covered with Parafilm to reduce evaporation.

For the study with intestinal flora, the operation was the same as described above for the study without intestinal flora except for the flush process.

Mesenteric blood was collected in heparinized centrifuge tubes within $1.5 \mathrm{~h}$. The blood samples were centrifuged at $5000 \mathrm{rpm}$ for $15 \mathrm{~min}$ to obtain the plasma, and then treated with 3 volumes of methanol to precipitate protein. The mixture was vortexed for $10 \mathrm{~min}$, and centrifuged at 10,000 rpm for $15 \mathrm{~min}$. The organic layer was transferred to another tube, and evaporated to dryness under a stream of nitrogen at $40^{\circ} \mathrm{C}$. Then, the dried residue was dissolved in $200 \mu \mathrm{L}$ of methanol for LC/MS analysis. 


\subsection{Gut Wall Metabolism Method}

About $10 \mathrm{~cm}$ of jejunum was selected and the surgical approach for metabolism without intestinal flora was used. Blood samples were collected and prepared as described above.

\subsection{Hepatic Metabolism Method}

The surgical approach was the same as described above but without blood supplementation and collection. Intestinal flora metabolism and gut wall metabolism would all occur before the hepatic metabolism, and thus $5 \mathrm{~cm}$ colon and $10 \mathrm{~cm}$ jejunum were used in this experiment. Blood samples were collected from the abdominal aorta after $1.5 \mathrm{~h}$ and prepared as described above.

\subsection{HPLC Analysis}

The HPLC system used to analyze the stability of salidroside in digestive juice was composed of a Waters 600 controller and pump and a Waters 2487 detector measuring absorbance at $214 \mathrm{~nm}$ (Waters Corporation, Milford, MA, USA). The chromatographic separation of salidroside was carried out on a $250 \mathrm{~mm} \times 4.6 \mathrm{~mm} \mathrm{C}_{18}$ column with acetonitrile/water 80:20 (v/v) as isocratic mobile phase at $1.0 \mathrm{~mL} / \mathrm{min}$. The column temperature was set at $35^{\circ} \mathrm{C}$ and the volume injected amounted to $20 \mu \mathrm{L}$.

\subsection{LC/MS Analysis}

Sample analyses were performed using an ultimate 3000 LC system coupled to an LTQ Orbitrapmass spectrometer via an ESI interface. The chromatography system consisted of an autosampler, a diode array detector, a column compartment and two pumps. Xcalibur, Metworks and Mass Frontier 7.0 software packages (Thermo Corporation, Waltham, MA, USA) were employed for data collection and data analysis.

Chromatographic separations were performed on a Thermo Scientific BOS Hypersil $\mathrm{C}_{18}$ column $2.1 \times 150 \mathrm{~mm}^{2}$, with $2.4 \mu \mathrm{m}$ particle size. The mobile phases A and B comprised $0.1 \%$ formic acid in water and acetonitrile, respectively. The LC gradient program (time (in min) $/ \%$ mobile phase B) was set as $0.01 / 1,3 / 1,6 / 5,12 / 10,19 / 50,24 / 85,26 / 1,30 / 1$. The chromatographic runs were performed at a flow rate of $0.3 \mathrm{~mL} / \mathrm{min}$. The injection volume was $3 \mu \mathrm{L}$ with column oven at $35^{\circ} \mathrm{C}$.

The ESI source parameters were as follows: the capillary temperature was $250^{\circ} \mathrm{C}$, source voltage and ispray voltage were set at $5 \mathrm{kV}$, shealth gas $\left(\mathrm{N}_{2}\right)$ flow was 35 psi. The ESI source was operated in the negative ionization mode. In the Fourier transform (FT) cell, full MS scans were acquired in the range of $m / z 50-1000$. The MS/MS experiments were set as data-dependent scans.

\section{Conclusions}

In this paper, a practical approach was established to study the sequential metabolism of salidroside after oral administration. Four metabolites were found in this sequential process and their structures were elucidated according to the retention times, accurate molecular mass, and characteristic fragment ions. The metabolic pathways were proposed and the relative contribution of each metabolic site to the total metabolism of the parent was also determined. Based on the result, the sequential process of salidroside after oral administration was clearly characterized. It was found that most salidroside was converted to the glucuronide and sulfate forms of both the parent compound and the aglycone, and then transported to the target tissues through the blood stream. The results obtained in this study could provide useful information about salidroside bioactivity and beneficial health effects. Furthermore, the proposed sequential metabolism methodology could be applicable to mechanistic studies of other compounds involving complicated sequential metabolic reactions.

Acknowledgments: This work was supported by the National Natural Science Foundation of China (30801510 and 81274042).

Author Contributions: Yang Liu conceived and designed the experiments; Zhiqiang Luo, Xiaoyun Ma, Lina Lu, Guohua Yu and Ruirui Yang performed the experiments; Zhiqiang Luo, Xiaoyun Ma and Mohan Sun analyzed 
the data; Shaokun Xin, Simin Tian, Xinjing Chen and Haiyu Zhao contributed reagents/materials/analysis tools; Zhiqiang Luo wrote the paper.

Conflicts of Interest: The authors declare no conflict of interest.

\section{References}

1. Yu, S.; Shen, Y.; Liu, J.; Ding, F. Involvement of ERK1/2 pathway in neuroprotection by salidroside against hydrogen peroxide-induced apoptotic cell death. J. Mol. Neurosci. 2010, 40, 321-331. [CrossRef] [PubMed]

2. Zhang, J.; Liu, A.; Hou, R.; Zhang, J.; Jia, X.; Jiang, W.; Chen, J. Salidroside protects cardiomyocyte against hypoxia-induced death: A HIF-1 $\alpha$-activated and VEGF-mediated pathway. Eur. J. Pharmacol. 2009, 607, 6-14. [CrossRef] [PubMed]

3. Li, M.; Dong, C.; Huai, L.; Bende, T.; Li, S.; Ying, W. Anti-fatigue effects of salidroside in mice. J. Med. Coll. PLA 2008, 23, 88-93. [CrossRef]

4. Liu, S.; Yu, X.; Hu, B.; Zou, Y.; Li, J.; Bo, L.; Deng, X. Salidroside rescued mice from experimental sepsis through anti-inflammatory and anti-apoptosis effects. J. Surg. Res. 2015, 195, 277-283. [CrossRef] [PubMed]

5. Díaz, L.A.; Abad, M.M.; Fernández, M.L.; Recuero, C.C.; Villaescusa, C.L.; Silván, S.A.; Bermejo, B.P. Lignan and phenylpropanoid glycosides from Phillyrea latifolia and their in vitro anti-inflammatory activity. Planta. Med. 2001, 67, 219-223. [CrossRef]

6. Zhang, L.; Yu, H.; Sun, Y.; Lin, X.; Chen, B.; Tan, C.; Cao, G.; Wang, Z. Protective effects of salidroside on hydrogen peroxide-induced apoptosis in SH-SY5Y human neuroblastoma cells. Eur. J. Pharmacol. 2007, 564, 18-25. [CrossRef] [PubMed]

7. Hu, X.; Lin, S.; Yu, D.; Qiu, S.; Zhang, X.; Mei, R. A preliminary study: The anti-proliferation effect of salidroside on different human cancer cell lines. Cell. Biol. Toxicol. 2010, 26, 499-507. [CrossRef] [PubMed]

8. Fang, D.; Chen, Y.; Xu, B.; Ren, K.; He, Z.; He, L.; Lei, Y.; Fan, C.; Song, X. Development of lipid-shell and polymer core nanoparticles with water-soluble salidroside for anti-cancer therapy. Int. J. Mol. Sci. 2014, 15, 3373-3388. [CrossRef] [PubMed]

9. Wang, J.; Li, J.; Lu, A.; Zhang, K.; Li, J. Anticancer effect of salidroside on A549 lung cancer cells through inhibition of oxidative stress and phospho-p38 expression. Oncol. Lett. 2014, 7, 1159-1164. [CrossRef] [PubMed]

10. Zhu, Y.; Shi, Y.; Wu, D.; Ji, Y.; Wang, X.; Chen, H.; Wu, S.; Huang, D.; Jiang, W. Salidroside protects against hydrogen peroxide-induced injury in cardiac H9c2 cells via PI3K-Akt dependent pathway. DNA. Cell. Biol. 2011, 30, 809-819. [CrossRef] [PubMed]

11. Adaptogen, A.P.P. Rhodiola rosea: A possible plant adaptogen. Altern. Med. Rev. 2001, 6, $293-302$.

12. Nan, J.; Jiang, Y.; Park, E.J.; Ko, G.; Kim, Y.C.; Sohn, D.H. Protective effect of Rhodiola sachalinensis extract on carbon tetrachloride-induced liver injury in rats. J. Ethnopharmacol. 2003, 84, 143-148. [CrossRef]

13. Zovko Koncic, M.; Tomczyk, M. New insights into dietary supplements used in sport: active substances, pharmacological and side effects. Curr. Drug. Targets. 2013, 14, 1079-1092. [CrossRef]

14. Skopińska-Rózewska, E.; Malinowski, M.; Wasiutyński, A.; Sommer, E.; Furmanowa, M.; Mazurkiewicz, M.; Siwicki, A. The influence of Rhodiola quadrifida $50 \%$ hydro-alcoholic extract and salidroside on tumor-induced angiogenesis in mice. Pol. J. Vet. Sci. 2007, 11, 97-104.

15. Fan, M.; Xu, S.; Xia, S.; Zhang, X. Preparation of salidroside nano-liposomes by ethanol injection method and in vitro release study. Eur. Food. Res. Technol. 2008, 227, 167-174. [CrossRef]

16. Wang, H.; Ding, Y.; Zhou, J.; Sun, X.; Wang, S. The in vitro and in vivo antiviral effects of salidroside from Rhodiola rosea L. against coxsackievirus B3. Phytomedicine 2009, 16, 146-155. [CrossRef] [PubMed]

17. Han, F.; Li, Y.; Mao, X.; Zhang, X.; Guan, J.; Song, A.; Yin, R. Metabolic profile of salidroside in rats using high-performance liquid chromatography combined with Fourier transform ion cyclotron resonance mass spectrometry. Anal. Bioanal. Chem. 2015, 408, 1975-1981. [CrossRef] [PubMed]

18. Hu, Z.; Wang, Z.; Liu, Y.; Wu, Y.; Han, X.; Zheng, J.; Yan, X.; Wang, Y. Metabolite Profile of Salidroside in Rats by Ultraperformance Liquid Chromatography Coupled with Quadrupole Time-of-Flight Mass Spectrometry and High-Performance Liquid Chromatography Coupled with Quadrupole-Linear Ion Trap Mass Spectrometry. J. Agr. Food. Chem. 2015, 63, 8999-9005. [CrossRef] [PubMed]

19. Guo, N.; Zhu, M.; Han, X.; Sui, D.; Wang, Y.; Yang, Q. The metabolism of salidroside to its aglycone $p$-tyrosol in rats following the administration of salidroside. PLoS ONE 2014, 9, e103648. [CrossRef] [PubMed] 
20. Zhang, L.; Zhao, H.; Liu, Y.; Dong, H.; Lv, B.; Fang, M.; Zhao, H. Metabolic routes along digestive system of licorice: multicomponent sequential metabolism method in rat. Biomed. Chromatogr. 2015, 30, 902-912. [CrossRef] [PubMed]

21. Ren, W.; Xin, S.; Han, L.; Zuo, R.; Li, Y.; Gong, M.; Wei, X.; Zhou, Y.; He, J.; Wang, H. Comparative metabolism of four limonoids in human liver microsomes using ultra-high-performance liquid chromatography coupled with high-resolution LTQ-Orbitrap mass spectrometry. Rapid. Commun. Mass. Spectrom. 2015, 29, 2045-2056. [CrossRef] [PubMed]

22. Zhang, Y.; Wu, W.; Han, F.; Chen, Y. LC/MS/MS for identification of in vivo and in vitro metabolites of jatrorrhizine. Biomed. Chromatogr. 2008, 22, 1360-1367. [CrossRef] [PubMed]

23. Luo, Z.; Liu, Y.; Zhao, B.; Tang, M.; Dong, H.; Zhang, L.; Lv, B.; Wei, L. Ex vivo and in situ approaches used to study intestinal absorption. J. Pharmacol. Toxicol. 2013, 68, 208-216. [CrossRef] [PubMed]

Sample Availability: Not available.

(C) 2016 by the authors; licensee MDPI, Basel, Switzerland. This article is an open access article distributed under the terms and conditions of the Creative Commons Attribution (CC-BY) license (http://creativecommons.org/licenses/by/4.0/). 